\title{
Avaliação do risco de quedas e qualidade de vida dos idosos com acidente vascular encefálico
}

\author{
Laila Cristina Moreira Damázio ${ }^{1}$ Jasiara Carla Oliveira² Elzira D’Santiago Marciano ${ }^{3}$ Marcos Grissi Pissolati ${ }^{4}$
}

\begin{abstract}
RESUMO
O presente estudo é randomizado pareado, cujo objetivo foi avaliar o risco de quedas em idosos com Acidente Vascular Encefálico (AVE), institucionalizados (grupo I) e não institucionalizados (grupo II). Os aspectos avaliados foram: capacidade funcional, equilíbrio corporal e qualidade de vida, por meio da escala de Dawton, escala de Medida de Independência Funcional (MIF), escala de Berg e o questionário The Medical Outcomes Study 36-item Short-Form Health Survey (SF-36), respectivamente. Ao avaliar o equilibrio e risco de quedas em ambos os grupos obteve-se diferença estatisticamente significativa, sendo que as médias para ambos foram favoráveis para os idosos do grupo II demonstrando que os idosos do grupo I estão mais propensos a quedas. Os resultados para análise da capacidade funcional também apresentaram diferença estatisticamente significativa, sendo que os idosos do grupo I obtiveram menor capacidade funcional. Conclui-se que idosos com sequela de AVE do grupo I apresentaram alto índice de quedas, além de menor capacidade funcional.
\end{abstract}

Descritores: Acidente Vascular Cerebral; Equilíbrio Postural; Idoso; Qualidade de vida.

\section{Assessment of risk of falls and quality of life of elderly patients with stroke}

\begin{abstract}
The present study is a randomized study that evaluated the risk of falls, functional capacity, body balance and quality of life through Dawton scale, scale of the Functional Independence Measure, scale of Berg, The Medical Outcomes Study 36-item short-Form Health Survey (SF-36), respectively. We evaluated the elderly with Stroke, 10 elderly subjects (group I) and 10 non-institutionalized elderly (group II). The assessment of balance and risk of falls in both groups yielded a statistically significant difference, the group II showed more risk to falls. The results for the functional analysis also showed statistically significant difference, and the elderly group I had lower functional capacity. In conclusion, elderly patients with sequelae of stroke in group I had lower balance providing a high rate of falls, and lower functional capacity.
\end{abstract}

Descriptors: Stroke; Postural balance; Aged; Quality of life.

\footnotetext{
${ }^{1}$ Doutoranda em Bioengenharia na Universidade Federal de São João Del-Rei (UFSJ), São João Del-Rei, MG, Brasil

${ }^{2}$ Especialista em fisioterapia respiratória pelo Biocor Instituto Biocor (Biocor), Nova Lima, MG, Brasil.

${ }^{3}$ Especialista em Psicopedagogia da educação pela Universidad de la Habana (UH), El Vedado, HAV, Cuba.

${ }^{4}$ Doutora em Biologia celular e estrutural pela Universidade Federal de Viçosa (UFV), Viçosa, MG, Brasil.
} 


\section{Introdução}

O envelhecimento é um processo normal, caracterizado pela diminuição da capacidade funcional dos diversos órgãos e pode ser visto tanto pelas alterações anatômicas, fisiológicas e psíquicas, quanto do ponto de vista moral e social ${ }^{1-2}$. A Organização Mundial da Saúde em suas projeções estatísticas expõe que, entre 1950 e 2025, a população de idosos no país crescerá 16 vezes enquanto que a população total crescerá apenas cinco vezes, o que nos colocará em termos absolutos com a sexta maior população de idosos do mundo, isto é, com mais de 32 milhões de pessoas com 60 anos ou mais ${ }^{3-4}$.

A qualidade de vida na terceira idade se vê ainda mais deteriorada quando estão presentes problemas de saúde, tais como Acidente Vascular Encefálico (AVE), que consequentemente aumenta a incidência de quedas, gerando incapacidades parciais ou dependência ${ }^{5}$. A incidência de AVE aumenta significativamente a partir dos 60 anos de idade ${ }^{6} \mathrm{e}$ apresenta manifestações clínicas que refletem a localização e extensão da lesão vascular. Pacientes com sequelas de AVE demonstram dificuldade em controlar o início do movimento bem como o controle motor voluntário ${ }^{7}$. Outras incapacidades também estão presentes, como paralisia de músculos, rigidez nas partes do corpo afetado, perda de mobilidades das articulações, dores difusas, problemas de memória, incapacidades sensoriais, dificuldade na comunicação oral e escrita, prejudicando a capacidade funcional ${ }^{4}$.

A incapacidade funcional aumenta os episódios de quedas que também desencadeiam impactos na vida de um idoso, como a hospitalização, institucionalização e consumo de serviços sociais e de saúde. No Brasil, em 2005, ocorreram 61.368 hospitalizações por quedas de pessoas idosas de ambos os sexos, representando 2,8\% de todas as internações de idosos no país5. A ocorrência de quedas em idosos que residem em instituições de longa permanência é bem maior se comparada à daqueles que residem com suas famílias na comunidade. Acredita-se que seja pelo fato desses idosos serem mais propensos ao sedentarismo ${ }^{8}$.

Desta forma, este estudo tem por avaliar o risco de quedas entre os idosos institucionalizados e não institucionalizados com sequelas de AVE por meio da capacidade funcional, equilíbrio corporal e qualidade de vida, permitindo averiguar qual grupo é mais debilitado e os fatores que mais contribuem para esse evento.

\section{Metodologia}

Trata-se de um estudo randomizado pareado no qual foram avaliados, o risco de quedas, equilíbrio corporal, capacidade funcional e qualidade de vida dos idosos com sequelas de AVE institucionalizados e não institucionalizados.

Os critérios de inclusão foram: indivíduos entre 60 e 80 anos diagnosticados com sequelas de AVE pelo serviço médico, obtidos pela leitura dos prontuários. Indivíduos com mais de 80 anos não foram incluídos no estudo por apresentarem maior dependência funcional, podendo interferir nos resultados ${ }^{9}$. Os critérios de exclusão foram indivíduos que apresentaram outras patologias neurológicas associadas, problemas cognitivos, problemas cardíacos e respiratórios graves, além de problemas ortopédicos que limitavam a deambulação e as funções de membros superiores causando dores.

A busca pela amostra de idosos institucionalizados foi realizada em três instituições de longa permanência na cidade de Barbacena-MG. Os idosos não institucionalizados foram avaliados em suas respectivas residências e em clínicas de fisioterapia da mesma cidade. A amostra final consistiu de 20 indivíduos, com sequelas de AVE, do gênero masculino (por oportunidade de amostra), divididos em dois grupos, sendo 10 indivíduos institucionalizados (Grupo I) e 10 não institucionalizados (Grupo II).

Para avaliação do equilíbrio corporal foi utilizada a escada de Berg (teste que contém 14 tarefas comuns que envolvem o equilíbrio estático e dinâmico). A realização das tarefas foi avaliada por meio de observação que resultou em pontuação de 0-4 (a pontuação aumenta conforme menor for a dependência para a realização das tarefas), totalizando um máximo de 56 pontos. Já a capacidade funcional foi realizada utilizando a Medida de Independência Funcional (MIF) que possui 18 categorias pontuadas de um a sete e classificadas quanto ao nível de dependência para a realização da tarefa.

O risco de quedas foi avaliado pela escala de Dawton que é dividida em cinco níveis, distribuídos em: 1 - quedas anteriores; 2 - medicamentos; 3 - déficit sensorial; 4 - estado mental e 5 - marcha. Os subníveis podem ser marcados com asteriscos, sendo que, cada asterisco significa um ponto. 0 risco elevado de queda pela escala de Dawton é estabelecido com um escore igual ou maior que 3 pontos. 
Para a avaliação da qualidade de vida foi utilizado o questionário genérico The Medical Outcomes Study 36-item Short-Form Health Survey (SF-36), validado na cultura brasileira, sendo considerado um instrumento simples, com questões diretas e de fácil compreensão ${ }^{10}$.

A coleta de dados foi realizada em duas etapas. A primeira etapa consistiu na seleção da amostra e a segunda etapa, na avaliação de todos os indivíduos com as escalas citadas anteriormente. Estes dados foram recolhidos e processados em banco de dados eletrônico no programa Microsoft ${ }^{\circledR}$ Excel 07 (Sistema Operacional Windows 7, Microsoft Coporation, Inc.). O teste estatístico utilizado foi o teste t-Student para amostras independentes com nível de significância de 5\%, e o teste de correlação de Pearson, com nível de significância de 5\%, para as correlações das variáveis em análise.

O estudo foi aprovado pelo Comitê de Ética e Pesquisa da Universidade Presidente Antônio Carlos - UNIPAC de Barbacena - Minas Gerais, (Parecer protocolo n 523/09), por estar de acordo com a Resolução de número $196 / 96$ do Conselho Nacional de Saúde para pesquisa com seres humanos. Os diretores das instituições de longa permanência e os idosos da comunidade receberam termo de consentimento livre e esclarecido, para a segurança dos participantes e da pesquisa, sendo o estudo iniciado após o consentimento dos mesmos.

\section{Resultados}

A faixa etária total dos idosos estudados variou de 61 a 77 anos, sendo que a média foi de $67,8 \pm 4,32$ para idosos institucionalizados (grupo I) e $66 \pm 4,19$ para idosos não institucionalizados (grupo II). Com relação ao tempo de ocorrência do AVE, o grupo I apresentou uma média de 39,6 meses, enquanto o grupo II apresentou média de 40,1 meses pós AVE.

Foram avaliados equilíbrio, risco de quedas, qualidade de vida e capacidade funcional de todos os idosos da amostra. A tabela 1 demonstra as médias com seus respectivos desvios padrões para as escala Berg, Dawton e MIF.

\begin{tabular}{|c|c|c|c|}
\hline & $\begin{array}{c}\text { Institucionalizados } \\
\text { Média } \pm \text { Desvio } \\
\text { Padrão (DP) }\end{array}$ & $\begin{array}{c}\text { Não institucionalizados } \\
\text { Média } \pm \text { Desvio } \\
\text { Padrão (DP) }\end{array}$ & $\mathrm{p}$-valor \\
\hline Berg & $37.000 \pm 3.2042$ & $46.400 \pm 1.6275$ & 0,0561 \\
\hline Dawton & $3.4000 \pm 0.58119$ & $1.2000 \pm 0.20000$ & 0,0040 \\
\hline MIF & $106.40 \pm 4.6000$ & $118.10 \pm 1.2152$ & 0,0005 \\
\hline
\end{tabular}

Os valores médios da Escala de Berg foram diferentes estatisticamente $(p<0,05)$, sendo que a média foi superior entre os idosos não institucionalizados quando comparado aos idosos institucionalizados (Figura 1). No entanto, de acordo com alguns autores, os valores encontrados para ambos os grupos de idosos se enquadram em um intervalo que sugere alto risco de queda devido ao déficit de equilíbrio ${ }^{11}$.

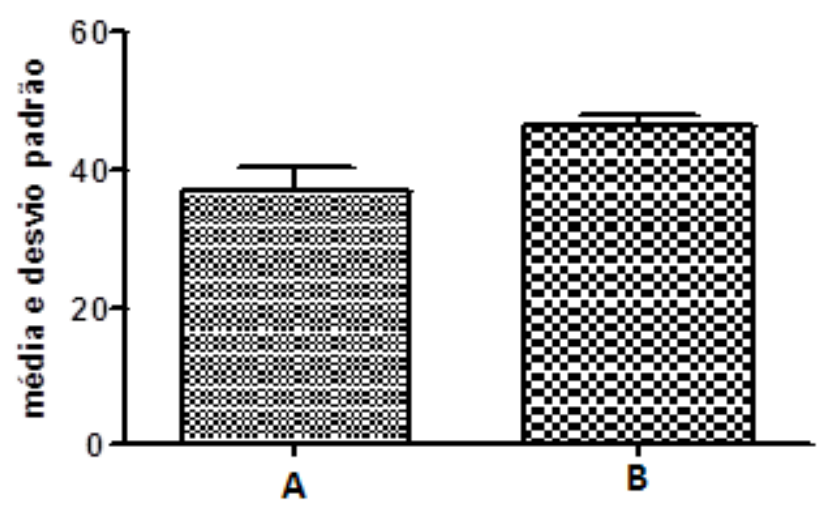

Figura 1 - Valores médios e desvio padrão do equilibrio corporal dos idosos com AVE institucionalizados (A) e não institucionalizados (B) avaliados pela escala de Berg. 
$\mathrm{Na}$ Escala de Dawton o valor médio foi desfavorável ao grupo de idosos institucionalizados que apresentaram uma média de escore de 3,4, ou seja, maior que três pontos, estando então, classificados no grupo de alto risco de quedas (Figura 2).

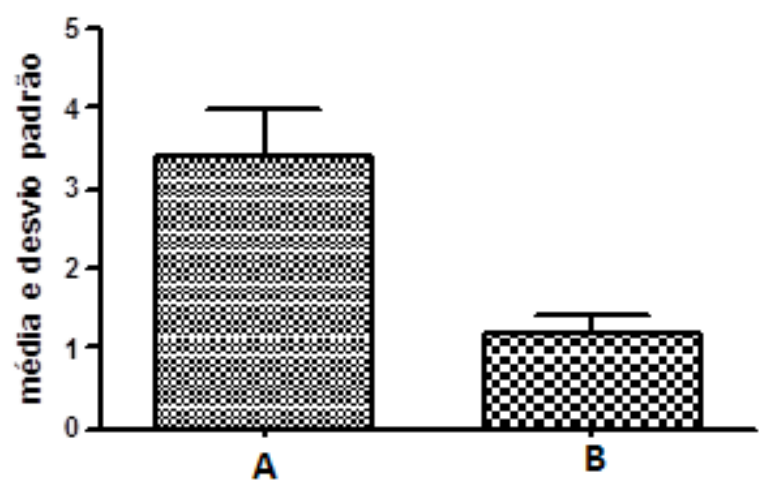

Figura 2 - Valores médios e desvio padrão do risco de quedas dos idosos com AVE institucionalizados (A) e não institucionalizados

(B) avaliados pela escala de Dawton.

Entre os itens avaliados da escala de Dawton, foi verificado o uso de medicamentos (tranquilizantes, diuréticos, hipotensores, antiparkinsonianos, antidepressivos e outros), em que cada grupo de medicamentos é equivalente a 1 ponto. $\mathrm{O}$ grupo I fazia uso de mais medicamentos $(1,5)$ que o grupo II $(0,9)$. Também foi analisada a qualidade da deambulação, classificando-a como: normal, segura com ajuda (pessoa ou dispositivo), insegura mesmo com ajuda ou impossível (uso de cadeira de rodas), correspondendo a 1 ponto cada item, exceto a deambulação normal. Novamente os idosos do grupo I apresentaram maior média $(0,6)$ quando comparados aos idosos do grupo II $(0)$, reservando ao primeiro, maior necessidade de ajuda para deambulação e maior risco de quedas.

O resultado para escala de MIF, que avalia capacidade funcional também foi estatisticamente significativo $(p<0,05)$, apresentando valor médio menor para idosos institucionalizados $(106,40)$ quando comparado aos idosos não institucionalizados $(118,10)$ (Figura 3), contudo ambos os valores médios se enquadram em uma independência funcional completa/modificada.

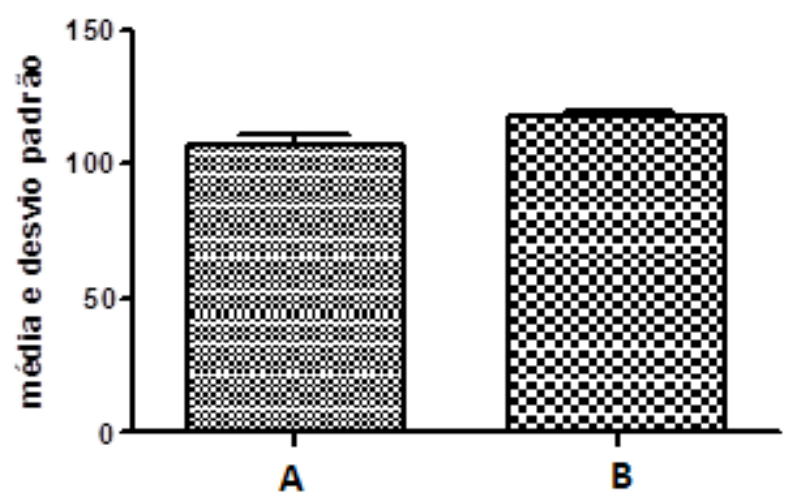

Figura 3 - Valores médios e desvio padrão da medida de independência funcional dos idosos com AVE institucionalizados (A) e não institucionalizados (B).

Os resultados da avaliação da qualidade de vida de todos os idosos foram comparados usando o questionário SF36 que apresenta oito domínios, sendo estes: capacidade funcional (CF), limitação por aspecto físico (LAF), dor (D), estado geral de saúde (EGS), vitalidade (V), aspecto social (AS), limitação por aspecto emocional (LAE) e saúde mental (SM). Para estes oito domínios, os valores médios dos itens CF, EGS, AS, LAE e SM foram superiores para idosos não institucionalizados, no entanto foi observada diferença estatisticamente significativa $(p<0,05)$, apenas para os domínios CF e LAE (Figura 4). 


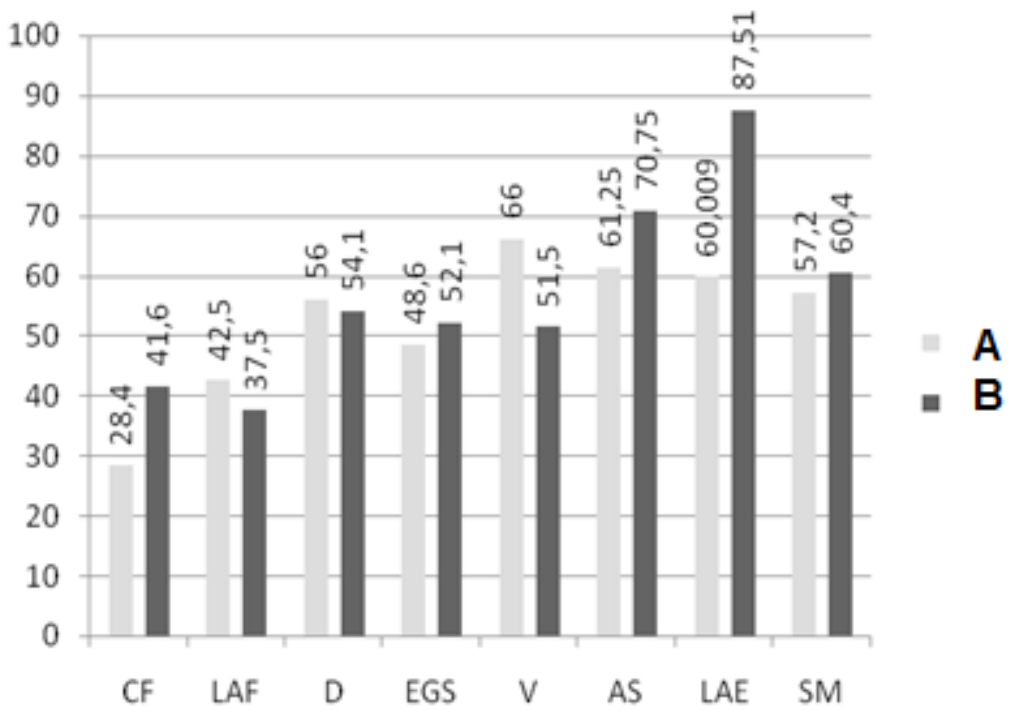

Figura 4 - Médias para os oito domínios do questionário SF 36 dos idosos com AVE institucionalizados (A) e não institucionalizados (B).

$\mathrm{Na}$ amostra pesquisada foi observada correlação significativa entre as escalas de Berg e Dawton (Pearson $r^{2}=-0,71$, $p=0,0002$ ), entre 0 risco de quedas e a capacidade funcional dos idosos (Pearson $r 2=0,63, p=0,003$ ), e entre 0 equilíbrio corporal e a capacidade funcional dos idosos (Pearson $r 2=0,67, p=0,001$ ).

\section{Discussão}

Como demonstrado houve diferença significativa $(p<0,05)$ entre as médias da escala de Berg de idosos com AVE, institucionalizados e não institucionalizados, sendo que os idosos institucionalizados apresentaram menor média na escala de Berg e MIF, e maior média na escala de Dawton. Isto corrobora com os estudos ${ }^{12-13}$ que evidenciaram maior incidência de quedas nos idosos institucionalizados devido à fragilidade e fraqueza muscular ${ }^{12}$. Esta ocorrência é maior pelo fato desses idosos apresentarem um longo período de sedentarismo ${ }^{13}$. Cerca de $50 \%$ das quedas têm como causa: ambiente inadequado, seguidos por doenças neurológicas e doenças cardiovasculares ${ }^{4}$.

Dentre os fatores intrínsecos responsáveis por quedas, está o surgimento de doenças que ocasionam redução da capacidade física podendo acarretar efeitos sobre o controle postural do indivíduo e ter ação sobre o equilíbrio. As principais condições patológicas que podem predispor às quedas são: as doenças neurológicas, cardiovasculares, endocrinológicas, osteomusculares, geniturinárias, psiquiátricas e sensoriais ${ }^{15-16}$.

No presente estudo, foi verificado que os idosos institucionalizados usavam maior quantidade de medicamentos e possuíam maior dificuldade para deambulação, o que os expunham a possíveis quedas. A correlação entre o risco de quedas e o uso de polifármacos está em concordância com o uso de quatro ou mais drogas associadas, em idosos, podendo ocasionar maior risco de queda devido à associação entre elas e ao fato de traduzir uma condição de saúde precária ${ }^{17}$. Medicações como diuréticos, psicotrópicos, anti-hipertensivos e antiparkinsonianos podem ser consideradas drogas que propiciam quedas. Estes medicamentos podem diminuir funções motoras, causar fraqueza muscular, fadiga, vertigem ou hipotensão postural ${ }^{18}$.

A senescência propicia desordens dos receptores sensoriais do equilíbrio, do controle postural, do processamento cognitivo central e da resposta motora, o que torna o indivíduo mais dependente ${ }^{19}$. Existe uma correlação entre quedas e capacidade funcional, onde o prejuízo da última tem papel importante na interação multicausal das quedas ${ }^{20}$. No presente estudo, ao avaliar a capacidade funcional de ambos os grupos de idosos foi observado que estes se enquadram em um nível de independência funcional completa/modificada. Todavia, os idosos do grupo I apresentaram menor escore na escala MIF, o que foi suficiente para proporcionar diferença estatística significativa entre os grupos. $O$ escore encontrado para o grupo de idosos com AVE de instituições foi de 106,40 classificando-os com dependência completa-modificada. Outro estudo ${ }^{21}$ que avaliou a capacidade funcional, através da escala MIF, de 14 idosos, com sequela de AVE, residentes em instituições de Goiânia encontrou um escore total de 60 (dependência modificada com assistência de 50\%). 0 fato de 
o idoso ter sofrido um AVE somado ao fato de ser institucionalizado pode justificar menor escore encontrado no presente estudo, pois o risco para o desenvolvimento de incapacidade funcional aumenta quando o indivíduo com sequela de AVE é idoso e institucionalizado22.

O fato dos idosos incluídos neste estudo apresentarem independência completa/modificada pode se justificar por todos possuírem acompanhamento em serviços de reabilitação como fisioterapia e terem mais de um ano de sequela de AVE, sugerindo que os idosos se adaptam com as incapacidades provocadas por esta patologia. Os idosos com seqüela de AVE que possuem acesso à assistência em saúde possuem maior escore na escala MIF. ${ }^{23}$ Já os indivíduos com sequela de AVE que têm idade igual ou superior a 55 anos apresentam dificuldade de autonomia, de independência e redução na capacidade funcional e que estes fatores quando associados repercutem no agravo da qualidade de vida ${ }^{24}$.

No presente estudo foram identificados valores médios superiores $(p<0,05)$ nos itens CF e LAE da escala SF-36 para os idosos não institucionalizados. Os domínios CF e LAF foram os itens que obtiveram menores escores tanto entre idosos institucionalizados quanto idosos não institucionalizados. Estes resultados corroboram achados do estudo ${ }^{25}$ que avaliou a qualidade de vida de idosos que vivem em residências, por meio do SF-36, com e sem assistência à saúde, que apontaram menor escore para os domínios aspecto físico, capacidade funcional e aspecto emocional. A presença de sequelas decorrentes do AVE, prejudica a funcionalidade dos idosos e também demonstra que os sujeitos com acesso à assistência à saúde apresentam escore superior na dimensão capacidade funcional, quando comparados aos que não possuem acesso ${ }^{25}$.

Além disso, as sequelas motoras, cognitivas, emocionais e sociais decorrentes do AVE, causam prejuízo no desempenho funcional e consequentemente na qualidade de vida dos idosos. Assim, a reabilitação é uma das principais formas de tratamento após um episódio de AVE proporcionando melhora e recuperação total ou parcial das funções comprometidas pela doença ${ }^{25}$.

A partir dos achados deste estudo, observa-se que idosos com sequelas de AVE institucionalizados (grupo I) apresentaram diminuição de equilíbrio proporcionando alto risco de quedas. Também apresentaram menor capacidade funcional quando comparados aos idosos, com sequelas de AVE, não institucionalizados (grupo II). No entanto, a qualidade de vida tanto no grupo I quanto no grupo II não apresentou diferenças estatísticas significativas.

\section{Conclusão}

Este estudo pode contribuir para a prática clínica, apontando dados que contribuem para melhorias na adequação das instituições de longa permanência de idosos com sequelas de AVE, além de proporcionar programas de manejo, promoção, prevenção e reabilitações específicas, para que o idoso tenha maior autonomia ao realizar suas atividades de vida diária.

\section{Referências bibliográficas}

1. Najas MS. A avaliação nutricional do Idoso. In: Papaléo Netto, editora. Atheneu. São Paulo; 1996. p. 242-247.

2. Gandolfi LM, Skora, MC. Fisioterapia preventiva em grupos da terceira idade. Fisioterapia em movimento. 2001; 13 (2): 55-62.

3. Ministério da Saúde. Redes Estaduais de Atenção à Saúde do Idoso: Guia Operacional e Portarias Relacionadas. [citado em: 12 out 2010]. Disponível em: http://portalsaude.saude.gov.br/portalsaude/index.cfm

4. Rabelo DF, Néri AL. Bem-estar subjetivo e senso de ajustamento psicológico em idosos que sofreram acidente vascular cerebral: uma revisão. Estudos de Psicologia. 2006; 11 (2): 169-177.

5. Ribeiro PA, Souza, ER. A influência das quedas na qualidade de vida de idosos. Rev. Ciência e Saúde Coletiva. 2008; 13(4): 1265-1273.

6. Paulo RB,Guimarães TM,Helito PVP,et al.Acidente vascular cerebral isquêmico em uma enfermaria de neurologia :complicações e tempo de internação. Rev Assoc Med Bras. 2009; 55(3): 313-316.

7. Salmela LF, Faria CDC. M., Guimarães, C. Q. Goulart, F. Parreira, V. F., Inácio, E. P. E Alcântara, T. O. Treinamento físico e destreinamento em hemiplégicos crônicos: impacto na qualidade de vida. Rev. Brasileira de Fisioterapia. 2005; 9(3): 347-353. 
8. Orondino JAS, Araújo CRM, Oliveira, EAT, Aguiar JLN. Incidência e causa de quedas em idoso institucionalizados. In: Anais do III Encontro de Iniciação Científica FAMINAS; 2006 jan.; Muriaé. Minas Gerais: 2006

9. Ricci NA, Kubota MT, Cordeiro RC. Concordância de observações sobre a capacidade funcional de idosos em assistência domiciliar. Rev. Saúde Pública. 2005; 39 (54): 655-62.

10. Ciconelli RM. Tradução para o português e validação do questionário genérico de avaliação da qualidade de vida. Medical Outcomes Study 36-item short-form health survey (SF-36) [Tese]. São Paulo: Universidade Federal de São Paulo, 1999.

11. Shumway-Cook A, Woollacott MH. Controle motor: teoria e aplicações práticas. $2^{\circ}$ Edição. Ed. Manole: 2003.

12. Díaz GJG, Gil CV, Andrade VP, Alonso, RP, Alvarez AS, Reinoso SH. Risk factors for falls amongst older people living in nursing home. Rev. Espanhola Geriatria e Gerontologia. 2009; 44(6): 301-304

13. Siqueira FV, Facchini LA, Piccini RX, Tomasi E, Thumé E, Silveira DS, et. al. Prevalência de quedas em idosos e fatores associados. Revista Saúde Pública. 2007; 41(5): 749-756.

14. Fabrício SCC, Rodrigues, RAP, Junior MLC. Causas e conseqüências de quedas de idosos atendidos em hospital público. Revista Saúde Pública. 2004; 38(1): 93-99.

15. Kay PD, Tideiksaar R. Quedas e distúrbios de marcha. In: ABRAMS W. B., BERKOW R. Manual Merck de Geriatria. São Paulo: Ed Roca; 1995.

16. Lipsitiz LA. An 85 years-old woman with a history of falls. The Journal of the American Medical Association. 1996; 276(1): 59-66

17. Robbins AS, Rubenstein LZ, Josephson KR, Schulman BL, Osterweil LD, Fine G. Predictors of falls among elderly people. Results of two population based studies. Archives of Internal Medicine. 1989; 149(7): 1628-1633.

18. Brito FC, Costa SMN. Quedas. In: Papaleo Netto M., Brito F. C. Urgências em geriatria, editora. Atheneu. São Paulo: 2001. p. 323-3235.

19. Menezes RL, Bachion RL. Estudo da presença de fatores de riscos intrínsecos para quedas, em idosos institucionalizados. Ciências \& Saúde Coletiva. 2008; 13(4): 1209-1218.

20. Cumming RG, Salked G, Thomas M, Szonyi G. Prospective study of the impact of fear of falling on activities of daily living, SF-36 scores, and nursing home admission. J Gerontol A Biol Sci Med Sc. 2000; 55(5): 299-305.

21. Viana FP, Lorenzo APC, Oliveira EF, Rezendes SM. Medida de independência funcional nas atividades de vida diária em idosos com seqüelas de acidente vascular encefálico no Complexo Gerontológico Sagrada Família de Goiânia. Rev. Brasileira de Geriatria e Gerontologia. 2008; 11(1): 17-28.

22. Silva F, Stroke I. Current aspects concerning prevention: We need to act. Medicina Interna. 2004; 11(2): 99-108.

23. Cruz KCT, Diogo MJE. Avaliação da capacidade funcional de idosos com acidente vascular encefálico. Acta Paulista de Enfermagem. 2009; 22(5): 666-672.

24. Cruz KCT. Avaliação da capacidade funcional e da qualidade de vida de indivíduos com acidente vascular encefálico com idade maior ou igual à 55 anos. [dissertação]. São Paulo: Universidade Estadual de Campinas; 2004.

25. Cruz KCT, Diogo MJE. Avaliação da capacidade funcional em idosos com Acidente Vascular Encefálico. Online Brazilian Journal of Nursing [serial online] 2008. [citado em: 15 Jun 2010]. Disponível em: http://www.uff.br/nepae/ siteantigo/otherissues.htm

\section{Laila Cristina Moreira Damázio}

Endereço para correspondência - Rua Dom Helvécio, 74. Bairro Dom Bosco, CEP 36301-160, São João Del Rei, MG, Brasil.

E-mail: lailacmdamazio@gmail.com

Lattes: http://lattes.cnpq.br/2543185250947429

Jasiara Carla Oliveira - jasi_oliveira@yahoo.com.br

Elzira D'Santiago Marciano - elziramarciano@yahoo.com.br

Marcos Grissi Pissolati - mgpissolati@gmail.com

Enviado em 21 de novembro de 2013.

Publicado em 07 de julho de 2014. 
